The book represents the proceedings of the IVth Erwin Baur Memorial Symposium held in 1966, and contains articles written by contributors from many countries. Although the Symposium was held in Germany, the articles are written in English throughout, except for two in French and the introductory address in German.

The contributions range from review-type articles such as that by Nilan on the nature of induced mutations and by Bergann on chimeral structure and instability, to the results and progress of practical plant improvement programmes representing an up to date survey of the world of mutation breeding in a number of crops. Since there are 54 contributors it is not possible to deal with each article individually, but a glance through the list of authors reveals the presence of many who are "household names" to the mutation breeder, and to quote Gustafsson in his general resumé: "The Symposium . . . reached . . . a high scientific standard. For natural reasons the lectures centred around plant species of economic importance but . . . covered a wide range of population dynamics."

Unlike the author of a book, the editor of Conference Proceedings can include only that which was presented by the contributors. A completely balanced exposition is not, therefore, likely. In this instance this is reflected, for example, in the relative paucity of information on improving quantitative characters and the absence of mention of some crop plants, although Gustafsson in his resumé helps to redress the balance by drawing attention to subjects not dealt with by previous authors.

The difficult task of editing this book has been well carried out leaving only small, not unpleasant, variations in style. The layout is neat and clear and the typography good with few minor errors; the numerous half-tone plates range from very good to adequate. Unfortunately, though, no doubt in the interests of cost of production, the thin card covers are not up to the task of protecting the contents nor do they present an attractive spine on the library shelf.

Thus, it appears that in spite of the limitations imposed by the fact that Induced Mutations and their Utilization is a symposium and not a completely balanced review of this field of work, it is nevertheless a useful publication and a companion to the previous volumes, especially Nos. I and II. Its usefulness lies in its bringing together examples of current mutation breeding projects and background information giving an awareness of the range of research going on in this field. By reading it the plant breeder may derive ideas applicable to his particular interests and is provided with a convenient source of up to date references for further reading. The geneticist also may derive ideas for worthy lines of investigation and would certainly obtain a wider appreciation of applied mutation breeding.

T. D. Johnston

Welsh Plant Breeding Station, Aberyswyth

\title{
A MATTER OF TECHNIQUE
}

HUMAN RADIATION CYTOGENETICS: Proceedings of an International Symposium held in Edinburgh, 12-15 October 1966. Edited by H. J. Evans, W. M. Court-Brown and A. S. McLean. North-Holland Publishing Company, 1967. Pp. 281. 70s.

The recent rapid developments in the field of human cytogenetics can be attributed almost directly to the technical refinements of the peripheral 
blood leukocyte culture system. This volume reports the proceedings of an International Symposium held in Edinburgh in October 1966 to discuss the application of these techniques to the study of radiation induced chromosome aberrations in human cells. The papers covered three main aspects of research activity in this field, namely, the results obtained with in vitro radiation, the role and survival times of lymphocytes following in vivo radiation exposure and the results obtained from studies on persons exposed to radiation in accident, or therapeutically or during the course of their normal occupation. Accordingly the layout of the paper in this volume follows this general plan, although the contents are in fact arranged in two parts which distinguish between in vitro and in vivo studies.

The papers included in Part I are, in the main, reports of studies involving in vitro radiation of peripheral blood. Following a brief account of the history and development of the leukocyte culture system by Dr P. Moorhead, the theme of the conference is set in a paper by Heddle, Evans and Scott. In this they draw attention to the complexity of the leukocyte culture system and the importance of standardising certain aspects of procedure, particularly fixation time, in order to minimise the errors which can arise from scoring mixed cell generations. The two papers which follow demonstrate these points in a convincing way and at the same time draw attention to some notable features of the radiation response of human chromosomes. Part I is concluded with two papers which envisage the automatic analysis of chromosome variation by computer, a development which is eagerly anticipated, judging from the remarks of other contributors.

It is clear that the work described in the first part of this volume contains much new and important information on the leukocyte culture system and its response to radiation. Nevertheless, it is generally agreed that a better understanding of the structure and dynamics of these cell populations is required as well as more information on the influence of the various technical procedures employed. A similar situation exists regarding the radiation response of leukocytes irradiated in vivo except that at this level there are even greater deficiencies in knowledge. This situation is remedied in part by the papers in Part 2 of this volume which attempt to assess the role of the small lymphocyte following irradiation in vivo, but again there are many unsolved problems. The incentives for solving these problems are considerable since one obvious goal of this work is the development of a biological dosimetric technique based on the amount of chromosome damage in persons exposed to radiation. The many difficulties which at present come between the realisation of this goal are evidenced by the later papers in this volume which report the results obtained from the culturing of leukocytes exposed to radiation in vivo.

The stated aim of this conference was to foster a greater awareness of the complexity of the peripheral leukocyte system both in culture and in vivo and the consequent need for refinement and standardisation of technical procedures. If the conference, and this resulting volume, achieve this much, then rapid and far-reaching developments can be expected in the future.

G. H. JonEs

Department of Genetics, University of Birmingham 